Article

\title{
Exact Solutions of Bernoulli and Logistic Fractional Differential Equations with Power Law Coefficients
}

\author{
Vasily E. Tarasov 1,2 (1) \\ 1 Skobeltsyn Institute of Nuclear Physics, Lomonosov Moscow State University, Moscow 119991, Russia; \\ tarasov@theory.sinp.msu.ru; Tel.: +7-495-939-59-89 \\ 2 Faculty of Information Technologies and Applied Mathematics, Moscow Aviation Institute \\ (National Research University), Moscow 125993, Russia
}

Received: 17 November 2020; Accepted: 14 December 2020; Published: 16 December 2020

\begin{abstract}
In this paper, we consider a nonlinear fractional differential equation. This equation takes the form of the Bernoulli differential equation, where we use the Caputo fractional derivative of non-integer order instead of the first-order derivative. The paper proposes an exact solution for this equation, in which coefficients are power law functions. We also give conditions for the existence of the exact solution for this non-linear fractional differential equation. The exact solution of the fractional logistic differential equation with power law coefficients is also proposed as a special case of the proposed solution for the Bernoulli fractional differential equation. Some applications of the Bernoulli fractional differential equation to describe dynamic processes with power law memory in physics and economics are suggested.
\end{abstract}

Keywords: Bernoulli differential equation; nonlinear fractional differential equation; fractional logistic equation; fractional dynamics; economic models with memory; fractional oscillator with memory

PACS: 45.10.Hj; 89.65.Gh

MSC: 26A33; 34A08; 91B55; 91B62; 34C15

\section{Introduction}

Fractional differential equations are equations with derivatives of arbitrary (integer and non-integer) positive orders [1-4]. These equations of non-integer orders can be used to describe processes with memory and non-locality in space and time in various sciences, including physics $[5,6]$, economics [7,8], and other sciences [9]. Nonlinear equations are an important type of fractional differential equation. Unfortunately, there are very few nonlinear fractional differential equations for which exact solutions are known, and there are significantly fewer of them than there are solutions for nonlinear differential equations of the integer order. Some examples of the exact analytical solutions of the nonlinear fractional differential equations are given in Section 4 of book [1] (see also [10-12]).

The nonlinear differential equation of the first order, which was proposed by Jacob Bernoulli in 1695 , is a well-known nonlinear equation that has exact solutions. One of the well-known special cases of the Bernoulli equation is the differential equation with quadratic non-linearity, which is called the logistic differential equation. The solution of the Bernoulli differential equation, which is a first-order differential equation, is considered in almost all textbooks on differential equations.

Unfortunately, an exact solution to the Bernoulli fractional differential equation (BFDE), which is not reducible to differential equations of the integer order, has not yet been proposed. In the mathematical literature, the Bernoulli fractional differential equation is considered only within the framework of numerical modeling. For example, the fractional generalization of the Bernoulli differential equation is 
considered in paper [13], where fractional derivatives of non-integer orders are used. This equation is solved numerically by using Newton's iteration method. Exact analytical solutions of this equation were not proposed in this article.

Logistic differential equations, which are a special case of the Bernoulli differential equation, are actively used in economics (for example, see $[14,15]$ ). Note also that the logistic differential equations with continuously distributed lag for exponential and gamma distributions of delay time and their application in economics was proposed in [16]. Note that fractional differential equations for the logistic growth model with memory [17] (see also [16]) and the model of logistic growth with memory and periodic sharp splashes (kicks) [18] describe economic processes with memory.

The special case of BFDE in the form of the fractional logistic differential equation (FLDE) is also considered only in the framework of numerical simulation. For the fractional logistic equation, a review of numerical solution methods is suggested in paper [19]. A possible expression of an exact solution of the fractional logistic equation with the Caputo fractional derivative was proposed by Bruce J. West [20] in the form of the Mittag-Leffler function. In paper [21], authors proved that the Mittag-Leffler function cannot be the solution for the FLDE. This fact is caused by the violation of the semi-group property by the Mittag-Leffler function. Therefore, the expression that is suggested by Bruce J. West in [20] cannot be a solution for the FLDE. At the present time, an exact analytical solution to the fractional logistic differential equation has not been obtained. We should note that the violation of the standard semi-group property, the violation of the standard product, and chain rules are important characteristics that should be taken into account in these equations. These non-standard properties significantly complicate the derivation of exact analytical solutions of nonlinear fractional differential equations and constructing mathematical models [17].

In this paper, the fractional generalization of the Bernoulli differential equation is considered. In this equation, we use the Caputo fractional derivative. We derive an exact solution of the special case of this nonlinear fractional differential equation and the conditions of the existence of solutions for this non-linear fractional differential equation. This special case is characterized by the power law form of coefficients. The proposed conditions impose restrictions on the existence of the solution for the non-linear fractional differential equation to describe processes with power law memory in applications. We also derive the exact solution of the fractional logistic differential equation with power law coefficients as a special case of the proposed solution for the Bernoulli fractional differential equation. Examples of applications of Bernoulli fractional differential equations in physics and economics are suggested.

\section{Nonlinear Fractional Differential Equation}

The standard Bernoulli differential equation is represented in the form

$$
X^{(1)}(t)+p(t) X(t)=q(t) X^{a}(t)
$$

where $a$ is a real number different from zero and one. For $a=0$ or $a=1$, we obtain an inhomogeneous or homogeneous linear differential equation. For $a=2$, the Bernoulli equation is a special case of the Riccati equation and the logistic differential equation. Usually, the logistic equation is considered with constant negative coefficients $p(t)=-r=$ const and $q(t)=-(r / K)=$ conts.

Let us consider the Bernoulli fractional differential equation that is represented in the form

$$
\left(D_{C ; t_{0}+}^{\alpha} X\right)(t)+p(t) X(t)=q(t) X^{a}(t),
$$

where $a>0, a \neq 1$, and $D_{C ; t_{0}+}^{\alpha}$ is the left-sided Caputo fractional derivative of the order $\alpha \geq 0$ [1] (p. 92). 
Definition 1. The Caputo fractional derivative is defined by the equation

$$
\left(D_{C ; t_{0}+}^{\alpha} X\right)(t)=\frac{1}{\Gamma(n-\alpha)} \int_{t_{0}}^{t} \frac{X^{(n)}(\tau) d \tau}{(t-\tau)^{\alpha-n+1}}
$$

where $n-1<\alpha \leq n, \Gamma(\alpha)$ is the gamma function, $t \in\left[t_{0}, t_{1}\right]$, and $X^{(n)}(\tau)$ is the integer-order derivative with $n \in \mathbb{N}$. It is assumed that $X(\tau) \in A C^{n}\left[t_{0}, t_{1}\right]$, i.e., the function $X(\tau)$, has derivatives $X^{(k)}(\tau)$ of orders $k=1,2, \ldots, n-1$, such that $X^{(k)}(\tau) \in C\left[t_{0}, t_{1}\right]$, and the derivative $X^{(n)}(\tau)$ is Lebesgue summable on the interval $\left[t_{0}, t_{1}\right]$.

For positive integer values $\alpha=n$, the Caputo derivative coincides (see Theorem 1 in [1] (p. 92)) with the standard derivative of integer order $n$, i.e., we have the equalities

$$
\left(D_{C ; t_{0}+}^{n} X\right)(t)=\frac{d^{n} X(t)}{d t^{n}},\left(D_{C ; t_{0}+}^{0} X\right)(t)=X(t)
$$

Therefore, for $\alpha=1$, Equation (2) takes form (1). For integer values of $\alpha=n$, Equation (2) takes form (1), where instead of the first-order derivative $X^{(1)}(t)$, there is an integer-order derivative $X^{(n)}(t)$.

We will consider the case of the power law form of the coefficients

$$
p(t)=p_{0}\left(t-t_{0}\right)^{\delta_{p}}, q(t)=q_{0}\left(t-t_{0}\right)^{\delta_{q}},
$$

where $t>t_{0}$. For $\delta_{p}=0$ and $\delta_{q}=0$, we get the case of the constant values of coefficients $p(t)=p_{0}=$ const and $q(t)=q_{0}=$ const, respectively.

Using function (5), the Bernoulli fractional differential equation takes the form

$$
\left(D_{C ; t_{0}+}^{\alpha} X\right)(t)=q_{0}\left(t-t_{0}\right)^{\delta_{q}} X^{a}(t)-p_{0}\left(t-t_{0}\right)^{\delta_{p}} X(t)
$$

where $t>t_{0}$. We would like to get an exact expression of the solution and the conditions of existence of this solution for nonlinear fractional differential Equation (6). To get the power law form of the solution, we will assume that $\delta_{q}=\delta$ and $\delta_{p}=-\alpha$.

We can state that Equation (6) for a wide class of functions and some ranges of parameter values has the solution in the form

$$
X(t)=C(\alpha)\left(t-t_{0}\right)^{\alpha-\gamma(\alpha)}
$$

where

$$
C(\alpha)=\left(\frac{p_{0} \Gamma(1-\gamma(\alpha))+\Gamma(\alpha-\gamma(\alpha)+1)}{q_{0} \Gamma(1-\gamma(\alpha))}\right)^{1 /(a-1)}
$$

and

$$
\gamma(\alpha)=\frac{\delta+a \alpha}{a-1}, \alpha-\gamma(\alpha)=\frac{\alpha+\delta}{1-a}
$$

To derive the exact solution and conditions of the existence of solution (7), we take into account the equation

$$
\left(D_{C, t_{0}+}^{\alpha}\left(\tau-t_{0}\right)^{b}\right)(t)=\frac{\Gamma(b+1)}{\Gamma(b-\alpha+1)}\left(t-t_{0}\right)^{b-\alpha},
$$

if $b>n-1$, and it is equal to zero if $b=0,1, \ldots n-1$. In all remaining cases $(b<n-1$, such that $b \neq 0,1, \ldots, n-1)$, the integral in the expression of the Caputo fractional derivative is improper and divergent. Equation (10) is given in book ([2], p. 49). Using Equation (10), it is directly verified that expression (7) is the explicit solution of Equation (6) for some ranges of parameter values, if $b=\alpha-\gamma(\alpha)>n-1$, where $n=[\alpha]+1$ for non-integer values of $\alpha>0$.

As a result, we can formulate the following theorem for nonlinear fractional differential Equation (6) with the Caputo fractional derivative of non-integer order. 
Theorem 1. The Bernoull fractional differential Equation (6) with $\alpha \in(n-1, n), n \in \mathbb{N}, \delta_{q}=\delta, \delta_{p}=-\alpha$, $q_{0} \neq 0$, and $a>0 t>t_{0}$ has a solution, which is given by Equations (7)-(9), if the conditions

$$
\left\{\begin{array} { l } 
{ a \in ( 0 , 1 ) } \\
{ \delta > - \alpha - ( n - 1 ) ( a - 1 ) , }
\end{array} \text { or } \left\{\begin{array}{l}
a>1, \\
\delta<-\alpha-(n-1)(a-1)
\end{array}\right.\right.
$$

are satisfied, where $q_{0}>0, p_{0}>0$ or

$$
\frac{p_{0} \Gamma(1-\gamma(\alpha))+\Gamma(\alpha-\gamma(\alpha)+1)}{q_{0} \Gamma(1-\gamma(\alpha))}>0 .
$$

This Theorem will be proved in the next section.

Remark 1. The conditions $q_{0}>0, p_{0}>0$, or inequality (12) can be omitted for even integer values of the parameter a.

\section{Conditions for Existence of an Exact Solution}

Let us give a proof of the proposed form for the explicit solution of the nonlinear fractional differential equation, and then we obtain conditions for the existence of this solution.

Let us consider the Bernoulli fractional differential equation

$$
\left(D_{C ; t_{0}+}^{\alpha} X\right)(t)=q_{0}\left(t-t_{0}\right)^{\delta_{q}} X^{a}(t)-p_{0}\left(t-t_{0}\right)^{\delta_{p}} X(t),
$$

where $t>t_{0}, \delta_{q}, \delta_{p} \in \mathbb{R}$, and $\alpha>0$ is non-integer positive parameter $\alpha \in(n-1, n), n \in \mathbb{N}$.

We will seek a solution in the power law form

$$
X(t)=C\left(t-t_{0}\right)^{b},
$$

where the coefficients $C>0$ and $b$ are real constants, and $t>t_{0}$. Substituting Equation (14) into fractional differential Equation (13), we obtain the equality

$$
C\left(D_{C ; t_{0}+}^{\alpha}\left(\tau-t_{0}\right)^{b}\right)(t)=q_{0}\left(t-t_{0}\right)^{\delta_{q}} C^{a}\left(t-t_{0}\right)^{a b}-p_{0}\left(t-t_{0}\right)^{\delta_{p}} C\left(t-t_{0}\right)^{b} .
$$

In the proof, we consider the following three cases: (A) the real values $b>n-1$; (B) the non-negative integer values $b \leq n-1$; and (C) the other real values $b \leq n-1$.

(A) For $b>n-1$, we should use the equation of the Caputo fractional derivative of the power law function in the form

$$
\left(D_{C, t_{0}+}^{\alpha}\left(\tau-t_{0}\right)^{b}\right)(t)=\frac{\Gamma(b+1)}{\Gamma(b-\alpha+1)}\left(t-t_{0}\right)^{b-\alpha}
$$

for non-integer values of $\alpha \in(n-1, n), n \in \mathbb{N}$. To get the power law form of the solution, we will assume that $\delta_{q}=\delta$ and $\delta_{p}=-\alpha$.

Using (16), Equation (15) takes the form

$$
C\left(p_{0}+\frac{\Gamma(b+1)}{\Gamma(b-\alpha+1)}\right)\left(t-t_{0}\right)^{b-\alpha}=q_{0}\left(t-t_{0}\right)^{\delta} C^{a}\left(t-t_{0}\right)^{a b}
$$

Using $C \neq 0$ and $t>t_{0}$, we get that Equation (17) holds, if the coefficients satisfy the conditions

$$
\left\{\begin{array}{l}
\frac{p_{0} \Gamma(b-\alpha+1)+\Gamma(b+1)}{\Gamma(b-\alpha+1)}=q_{0} C^{a-1}, \\
b-\alpha=\delta+a b .
\end{array}\right.
$$


Assuming that the inequality

$$
\frac{p_{0} \Gamma(b-\alpha+1)+\Gamma(b+1)}{q_{0} \Gamma(b-\alpha+1)}>0
$$

is satisfied for $a>0(a \neq 1)$, we obtain

$$
\left\{\begin{array}{l}
C=\left(\frac{p_{0} \Gamma(b-\alpha+1)+\Gamma(b+1)}{q_{0} \Gamma(b-\alpha+1)}\right)^{1 /(a-1)} \\
b=\frac{\delta+\alpha}{1-a} .
\end{array}\right.
$$

Here we see that the conditions in the form $q_{0}>0, p_{0}>0$ or inequality (12) can be omitted for even integer values of the parameter $a$.

Therefore, function (14) takes the form

$$
X(t)=\left(\frac{p_{0} \Gamma(b-\alpha+1)+\Gamma(b+1)}{q_{0} \Gamma(b-\alpha+1)}\right)^{1 /(a-1)}\left(t-t_{0}\right)^{(\delta+\alpha) /(1-a)} .
$$

and can be considered a solution of fractional differential Equation (13), with $\delta_{p}=-\alpha, \delta_{q}=\delta$ under some conditions (these conditions will be described below).

To simplify expressions, we will use the notation

$$
\gamma(\alpha)=\frac{\delta+a \alpha}{a-1}, \alpha-\gamma(\alpha)=\frac{\delta+\alpha}{1-a}
$$

(B) For integer values $b \leq n-1$, i.e., $b=0,1,2, \ldots, n-1$, we should use the equality

$$
\left(D_{C, t_{0}+}^{\alpha}\left(\tau-t_{0}\right)^{b}\right)(t)=0
$$

for non-integer values of $\alpha \in(n-1, n), n \in \mathbb{N}$. Substituting Equation (23) into fractional differential Equation (13), we obtain the equality

$$
q_{0}\left(t-t_{0}\right)^{\delta} C^{a}\left(t-t_{0}\right)^{a b}=C p_{0}\left(t-t_{0}\right)^{b-\alpha}
$$

Equality (24) gives the condition of existence of the solution for Equation (24), with non-zero values of the parameters, $q_{0}$, and $t>t_{0}$, in the form

$$
\left\{\begin{array}{l}
C=\left(\frac{p_{0}}{q_{0}}\right)^{1 /(a-1)} \\
b=\frac{\delta+\alpha}{1-a}
\end{array}\right.
$$

(C) For $b \leq n-1$ such that $b \neq 0,1, \ldots, n-1$, the integral in the expression of the Caputo fractional derivative is improper and divergent.

As a result, solution (21) takes the form

$$
X(t)=\left(\frac{p_{0} \Gamma(1-\gamma(\alpha))+\Gamma(\alpha-\gamma(\alpha)+1)}{q_{0} \Gamma(1-\gamma(\alpha))}\right)^{1 /(a-1)}\left(t-t_{0}\right)^{\alpha-\gamma(\alpha)}
$$

where $a>0, a \neq 1, q_{0} \neq 0, b>n-1, \alpha \in(n-1, n)$, and $n \in \mathbb{N}$. By definition of the Caputo fractional derivative, solution (26) must be continuous and hence bounded in a right neighborhood of $t_{0}$, i.e., $\left[t_{0}, t_{0}+\varepsilon\right]$ with $\varepsilon>0$, which yields the condition $\alpha-\gamma(\alpha) \geq 0$, and hence $X(t) \in C\left[t_{0}, t_{1}\right]$.

Using expression (26), the right-hand side of Equation (13) has the form

$$
F[t, X(t)]=q(t) X^{a}(t)-p(t) X(t)=C_{F}(\alpha)\left(t-t_{0}\right)^{a(\alpha-\gamma(\alpha))+\delta} .
$$


Here

$$
\begin{gathered}
C_{F}(\alpha)=q_{0}\left(\frac{p_{0} \Gamma(1-\gamma(\alpha))+\Gamma(\alpha-\gamma(\alpha)+1)}{q_{0} \Gamma(1-\gamma(\alpha))}\right)^{a /(a-1)} \\
\quad-p_{0}\left(\frac{p_{0} \Gamma(1-\gamma(\alpha))+\Gamma(\alpha-\gamma(\alpha)+1)}{q_{0} \Gamma(1-\gamma(\alpha))}\right)^{1 /(a-1)},
\end{gathered}
$$

where inequality (12) holds for $a>0$.

Using equality (22), we get

$$
a(\alpha-\gamma(\alpha))+\delta=a\left(\alpha-\frac{\delta+a \alpha}{a-1}\right)+\delta=-\frac{\delta+a \alpha}{a-1}=-\gamma(\alpha) .
$$

Therefore, expression (27) takes the form

$$
F[t, X(t)]=q(t) X^{a}(t)-p(t) X(t)=C_{F}(\alpha)\left(t-t_{0}\right)^{-\gamma(\alpha)} .
$$

The right-hand side of Equation (13), which is given by expression (30), belongs to $C_{\gamma}\left[t_{0}, t_{1}\right]$ in the case $\gamma(\alpha) \in(0,1)$, and function (30) belongs to $C\left[t_{0}, t_{1}\right]$ in the case $\gamma(\alpha) \leq 0$. Therefore, we have condition $\gamma(\alpha)<1$. Here, $C_{\gamma}\left[t_{0}, t_{1}\right]$ is the weighted space of functions $X(\tau)$ given on $\left(t_{0}, t_{1}\right]$, such that the function $\left(\tau-t_{0}\right)^{\gamma} A(\tau) \in C\left[t_{0}, t_{1}\right]$, and

$$
\|X(\tau)\|_{C_{\gamma}}=\left\|\left(\tau-t_{0}\right)^{\gamma} X(\tau)\right\|_{C}, C_{0}\left[t_{0}, t_{1}\right]=C\left[t_{0}, t_{1}\right] .
$$

As a result, we have the conditions of the existence of solution (26) by the system of inequalities

$$
\left\{\begin{array}{l}
a>0, a \neq 1 \\
\alpha \in(n-1, n) \\
\gamma(\alpha)<1 \\
b=\alpha-\gamma(\alpha)>n-1
\end{array}\right.
$$

The conditions $b>n-1$ of system (32) have the form $\alpha-\gamma(\alpha)>n-1$. Then we have $\gamma(\alpha)<\alpha-(n-1)$ that can be written as $\gamma(\alpha)<\{\alpha\}$, where $\{\alpha\} \in(0,1)$ is the fractional part of $\alpha$, i.e., $\alpha=[\alpha]+\{\alpha\}$, and $[\alpha]=n-1$. Therefore, if the condition $b=\alpha-\gamma(\alpha)>n-1$ holds, then inequality $\gamma(\alpha)<1$ is satisfied. This fact allows us to represent system (32) in the form

$$
\left\{\begin{array}{l}
a>0, a \neq 1 \\
\alpha \in(n-1, n) \\
b=\frac{\delta+\alpha}{1-a}>n-1
\end{array}\right.
$$

As a result, we have the following two cases

$$
\left\{\begin{array} { l } 
{ a \in ( 0 , 1 ) , } \\
{ \alpha \in ( n - 1 , n ) , } \\
{ \delta + \alpha > ( n - 1 ) ( 1 - a ) , }
\end{array} \text { or } \left\{\begin{array}{l}
a>1, \\
\alpha \in(n-1, n) \\
\delta+\alpha<(n-1)(1-a)
\end{array}\right.\right.
$$

These systems give conditions (11) that are used in Theorem 1 . The solution of nonlinear fractional differential Equation (13) with $\alpha \in(n-1, n)$ and $n \in \mathbb{N}$ is described by Equations (26) if conditions (34) are satisfied. This ends the proof. Q.E.D.

\section{Fractional Logistic Differential Equation with Power Law Coefficients}

The first time the logistic differential equation (LDF) was suggested was by Pierre F. Verhulst in 1845. Verhulst's model describes the growth of a population. This LDF is actively applied in economics (for example, see [14,15]). The LDF can be considered as a special case of the equation of the economic model of natural growth in a competitive environment [22] (pp. 84-90). The logistic equation with 
continuously distributed lag and its application in economics is given in [16]. Natural growth in a competitive environment with memory was first proposed in article [18].

Let us consider the fractional logistic differential equation in the form

$$
\left(D_{C ; t_{0}+}^{\alpha} X\right)(t)=r(t) X(t)\left(1-K^{-1}(t) X(t)\right)
$$

where $D_{C ; t_{0}+}^{\alpha}$ is the Caputo fractional derivative (3). Equation (35) is a special form of the Bernoulli fractional differential Equation (2), where $a=2, p(t)=-r(t)$, and $q(t)=-r(t) K^{-1}(t)$.

We will consider the case of the power law form of the coefficients

$$
r(t)=r_{0}\left(t-t_{0}\right)^{\delta_{r}}, K(t)=K_{0}\left(t-t_{0}\right)^{\delta_{K}},
$$

where $t>t_{0}$. For $\delta_{r}=0, \delta_{K}=0$, and $\alpha=1$, we get the standard form of the logistic differential equation of the first order with constant coefficients.

Using Theorem 1, we can formulate the following corollary.

Corollary 1. The fractional logistic differential Equation (35) with coefficients (36), where $\alpha \in(n-1, n), n \in \mathbb{N}$, $\delta_{r}=-\alpha, r_{0}, K_{0} \neq 0$, and $t>t_{0}$, has the solution in the form

$$
X(t)=C_{L}(\alpha)\left(t-t_{0}\right)^{\delta_{K}}
$$

where

$$
C_{L}(\alpha)=K_{0} \frac{r_{0} \Gamma\left(\delta_{K}+1-\alpha\right)-\Gamma\left(\delta_{K}+1\right)}{r_{0} \Gamma\left(\delta_{K}+1-\alpha\right)} .
$$

This solution exists if the condition

$$
\delta_{K}>(n-1)
$$

is satisfied.

The proof of this corollary follows directly from the proof of Theorem 1, where we take into account the values of the parameters $a=2, p(t)=-r(t), q(t)=-r(t) K^{-1}(t), p_{0}=-r_{0}, q_{0}=-r_{0} K_{0}^{-1}$, and $\delta=-\alpha-\delta_{K}$. In this case, $\gamma(\alpha)=\alpha-\delta_{K}$, and $\alpha-\gamma(\alpha)=\delta_{K}$.

\section{Application in Physics and Economics}

Let us consider some examples of an application of the Bernoulli fractional differential equation in physics and economics.

The first example is related to physics, or rather to an oscillator with memory [23-27] (see also [5]). A nonlinear one-dimensional oscillator without memory and dissipation is described by the equation of motion

$$
m X^{(2)}(t)=F(t, X(t))=-\frac{\partial U(t, x(t)}{\partial X(t)}
$$

where $U(t, X(t))$ is the time-dependent potential energy and $m$ is the mass of the particle. Here, $X(t)$ is the deviation of the point-particle from the equilibrium position.

If we assume that the potential energy is described by the expression

$$
U(t, X(t))=\frac{k(t) X^{2}(t)}{2}-\frac{b(t) X^{a+1}(t)}{a+1},
$$

where $a \neq-1$, then Equation (40) has the form

$$
m X^{(2)}(t)=-k(t) X(t)+b(t) X^{a}(t)
$$


As a result, the nonlinear oscillator is described by the differential equation

$$
X^{(2)}(t)+p(t) X(t)=q(t) X^{a}(t)
$$

where $p(t)=\omega^{2}(t)=k(t) / m$ and $q(t)=b(t) / m$.

A nonlinear one-dimensional oscillator with power law memory and the potential energy (41) is described by the equation

$$
\left(D_{C ; t_{0}+}^{\alpha} X\right)(t)+p(t) X(t)=q(t) X^{a}(t)
$$

where $p(t)=\omega^{2}(t)=k(t) / m$ and $q(t)=b(t) / m$. Equation (44) is the Bernoulli fractional differential equation. If $\alpha=2$, then Equation (44) takes the form of (43).

The second example is related to economics, or rather to the Solow model of economic growth $[22,28,29]$. The standard Solow model with continuous time is described by the nonlinear ordinary differential equation

$$
k^{(1)}(t)=s A k^{a}(t)-(\rho+\eta) k(t)
$$

where $k(t)$ is the per capita capital; $s$ is the rate of accumulation; $A$ is effectiveness of labor (knowledge) [29] (p. 9); $\rho$ is the rate of increase in labor resources; and $\eta$ is the capital retirement ratio. Here, it is assumed that the function of labor productivity is considered in the standard form $f(k(t))=A k^{a}(t)$, with $a \in(0,1)$.

In general, the parameters of the model can depend on time. As a result, the standard Solow model of economic growth is described by the nonlinear differential equation of the first order in the form

$$
X^{(1)}(t)=q(t) X^{a}(t)-p(t) X(t),
$$

where $X(t)=k(t)$ is the per capita capital, $q(t)=s(t) A(t)$ and $p(t)=\rho(t)+\eta(t)$. Note that the knowledge $A=A(t)$ is often viewed as a function of time in economic models [29] (p. 9).

A nonlinear economic model of growth with power-law memory is described by the fractional differential equation

$$
\left(D_{C ; t_{0}+}^{\alpha} X\right)(t)=-p(t) X(t)+q(t) X^{a}(t),
$$

where $X(t)=k(t)$ is the per capita capital, $q(t)=s(t) A(t)$ and $p(t)=\rho(t)+\eta(t)$. Equation (47) is the Bernoulli fractional differential equation. If $\alpha=1$, then Equation (47) takes the form of (46). Note that the proposed Solow model with memory can be considered as a generalization of the model that is suggested in [12].

The third example is connected with natural growth in a competitive environment (NGICE), which is used in economics [18,22]. For a linear price function, the NGICE model gives the model of logistic growth [22], which is described by the logistic differential equation.

Les us consider the economic model of natural growth in a competitive environment with power law memory (for example, see [18]). In this case, the function $Y(t)$ describes the value of output at time $t$. To describe the investments, which are made in the expansion of production, we will use the function $I(t)$. The main assumptions, which are used in the standard model of natural growth in a competitive environment without memory, are the following.

The first assumption is that the rate of change in output $\left(Y^{(1)}(t)\right)$ is proportional to the value of the investment $I(t)$. This assumption is represented by the accelerator equation

$$
I(t)=v Y^{(1)}(t)
$$

where $v$ is the accelerator coefficient (investment coefficient) that indicates the power of the economic accelerator. 
The second assumption is that the amount of investment $I(t)$ is a fixed part of the income $P Y(t)$, which is represented by the equation of linear multiplier

$$
I(t)=m P Y(t)
$$

where $m$ is the norm of net investment. This coefficient indicates the share of income that is spent to expand production.

The third assumption is that the price $P(t)$ depends on output $Y(t)$ that is considered as released product, i.e., $P=P(Y(t))$. Usually, we consider the case when the price decreases with increasing output, i.e., $P=P(Y)$ is a decreasing function. In a simple form, the price is a linear function of output $Y(t)$. In general, we can use the expression

$$
P(Y(t))=b-c Y^{a-1}(t),
$$

where $b$ is the price independent of $Y, a$ is the parameter characterizing deviation from linearity, and $c$ is interpreted as the marginal price if $a=2$. For $a=2$, Equation (50) is linear. The linear form of the function $P=P(Y)$ is often obtained by using the standard Taylor series with respect to $Y$. Expression (50) can be obtained by using the fractional Taylor series in the Odibat-Shawagfeh form [30], which is used for the function $P=P(Y)$.

In general, the parameters of the model can depend on time. Substituting (49) and (50) into Equation (48), we obtain

$$
Y^{(1)}(t)=\frac{m(t)}{v(t)} P(Y(t)) Y(t)=\frac{m(t) b(t)}{v(t, \alpha)} Y(t)-\frac{m(t) c(t)}{v(t, \alpha)} Y^{a}(t) .
$$

Equation (51) describes the standard NGICE model without memory. Note that the analogous type of nonlinear equation is used in the standard Haavelmo cyclic growth model [31].

To take into account the power law memory, the fractional derivatives of the non-integer order can be used instead of the first-order derivative in Equations (48) and (50). In this case, we obtain the nonlinear fractional differential equation

$$
\left(D_{C ; t_{0}+}^{\alpha} Y\right)(t)=\frac{m(t)}{v(t, \alpha)} P(Y(t)) Y(t)=\frac{m(t) b(t)}{v(t, \alpha)} Y(t)-\frac{m(t) c(t)}{v(t, \alpha)} Y^{a}(t)
$$

where $\left(D_{C ; t_{0}+}^{\alpha} Y\right)(t)$ is the Caputo derivative (3).

As a result, the model of natural growth in a competitive environment with power law memory is described by the Bernoulli fractional differential equation of the order $\alpha>0$ in the form

$$
\left(D_{C ; t_{0}+}^{\alpha} Y\right)(t)=q(t) Y^{a}(t)-p(t) Y(t),
$$

where $q(t)=-m(t) c(t) / v(t, \alpha)$ and $p(t)=-m(t) b(t) / v(t, \alpha)$.

Equation (53) describes the model of natural growth in a competitive environment with memory, which takes into account the memory with power law fading. For $\alpha=1$, Equation (53) gives standard Equation (51), which describes the standard NGICE model without memory. For $a=2$, Equation (53) is the fractional logistic differential equation that describes the logistic growth with memory. For $\alpha=1$ and $a=2$, Equation (53) gives the standard LDE that describes the standard model of economic growth without memory.

Funding: No external funding for this research.

Conflicts of Interest: The author declares no conflict of interest. The funders had no role in the design of the study; in the collection, analyses, or interpretation of data; in the writing of the manuscript, or in the decision to publish the results. 


\section{References}

1. Kilbas, A.A.; Srivastava, H.M.; Trujillo, J.J. Theory and Applications of Fractional Differential Equations; Elsevier: Amsterdam, The Netherlands, 2006; 540p, ISBN 978-0-444-51832-3.

2. Diethelm, K. The Analysis of Fractional Differential Equations: An Application-Oriented Exposition Using Differential Operators of Caputo Type; Springer: Berlin, Germany, 2010; 247p. [CrossRef]

3. Kochubei, A.; Luchko, Y. Handbook of Fractional Calculus with Applications. Basic Theory; Walter de Gruyter GmbH: Berlin, Germany; Boston, MA, USA, 2019; Volume 1, 481p, ISBN 978-3-11-057081-6. [CrossRef]

4. Kochubei, A.; Luchko, Y. Handbook of Fractional Calculus with Applications. Fractional Differential Equations; Walter de Gruyter GmbH: Berlin, Germany; Boston, MA, USA, 2019; Volume 2, 519p, ISBN 978-3-11-057082-3. [CrossRef]

5. Tarasov, V.E. Handbook of Fractional Calculus with Applications. Volume 4. Application in Physics. Part A; Walter de Gruyter GmbH: Berlin, Germany; Boston, MA, USA, 2019; 306p, ISBN 978-3-11-057088-5. [CrossRef]

6. Tarasov, V.E. Handbook of Fractional Calculus with Applications. Volume 5. Application in Physics. Part B; Walter de Gruyter GmbH: Berlin, Germany; Boston, MA, USA, 2019; 319p, ISBN 978-3-11-057089-2. [CrossRef]

7. Tarasov, V.E. Mathematical Economics: Application of Fractional Calculus; MDPI: Basel, Switzerland; Beijing, China, 2020; 278p, ISBN 978-3-03936-118-2/978-3-03936-119-9. Available online: https://www.mdpi.com/ books/pdfview/book/2350 (accessed on 12 December 2020). [CrossRef]

8. Tarasov, V.E.; Tarasova, V.V. Economic Dynamics with Memory: Fractional Calculus Approach; De Gruyter: Berlin, Germany; Boston, MA, USA, 2021; 576p, Hardcover ISBN 978-3-11-062460-1; PDF ISBN 978-3-11-062745-9 EPUB ISBN 978-3-11-062481-6. Available online: https://www.degruyter.com/view/title/542493 (accessed on 12 December 2020).

9. Baleanu Lopes, D.A.M.; Tenreiro Machado, J.A. (Eds.) Handbook of Fractional Calculus with Applications. Volume 8: Applications in Engineering, Life and Social Sciences, Part B; De Gruyter: Berlin, Germany; Boston, MA, USA, 2019; ISBN 978-3-11-057092. [CrossRef]

10. Tarasov, V.E. Fractional nonlinear dynamics of learning with memory. Nonlinear Dyn. 2020, 100, 1231-1242. [CrossRef]

11. Tarasov, V.E. Corrigendum to "Fractional nonlinear dynamics of learning with memory". Nonlinear Dyn. 2020, 100, 1231-1242, Nonlinear Dyn. 2021, accepted for publication 2020.12.02.

12. Tarasov, V.E. Non-linear macroeconomic models of growth with memory. Mathematics 2020, 8, 2078. [CrossRef]

13. Myshkin, S.V. On one model integral-differential Bernull equation. Bull. KRASEC Phys. Math. Sci. 2017, 2, 59-64. Available online: http://mi.mathnet.ru/eng/vkam199 (accessed on 12 December 2020). [CrossRef]

14. Kwasnicki, W. Logistic growth of the global economy and competitiveness of nations. Technol. Forecast. Soc. Chang. 2013, 80, 50-76. Available online: http://kwasnicki.prawo.uni.wroc.pl/todownload/ WKLogisticGrowthTF\&SChFinal.pdf (accessed on 12 December 2020). [CrossRef]

15. Ferrara, M.; Guerrini, L. The neoclassical model of Solow and Swan with logistic population growth. In Proceedings of the 2nd International Conference of IMBIC on Mathematical Sciences for Advancement of Science and Technology (MSAST), Kolkata, India, 21-23 December 2017; pp. 119-127. Available online: https:// www.unirc.it/documentazione/materiale_didattico/600_2008_108_1691.pdf (accessed on 12 December 2020).

16. Tarasov, V.E.; Tarasova, V.V. Logistic equation with continuously distributed lag and application in economics. Nonlinear Dyn. 2019, 97, 1313-1328. [CrossRef]

17. Tarasov, V.E. Rules for fractional-dynamic generalizations: Difficulties of constructing fractional dynamic models. Mathematics 2019, 7, 554. [CrossRef]

18. Tarasova, V.V.; Tarasov, V.E. Logistic map with memory from economic model. Chaos Solitons Fractals 2017, 95, 84-91. [CrossRef]

19. Saad, K.M.; Al-Shomrani, A.A.; Mohamed, M.S.; Yang, X.J. Solving fractional order logistic equation by approximate analytical methods. Int. J. Open Probl. Comput. Sci. Math. 2016, 9, 3. Available online: http://www.i-csrs.org/Volumes/ijopcm/vol.9/9.2.3.pdf (accessed on 12 December 2020).

20. West, B.J. Exact solution to fractional logistic equation. Phys. A Stat. Mech. Its Appl. 2015, 429, $103-108$. [CrossRef]

21. Area, I.; Losada, J.; Nieto, J.J. A note on the fractional logistic equation. Phys. A Stat. Mech. Its Appl. 2016, 444, 182-187. [CrossRef] 
22. Volgina, O.A.; Golodnaya, N.Y.; Odiyako, N.N.; Shuman, G.I. Mathematical Modeling of Economic Processes and Systems, 3rd ed.; Knorus: Moscow, Russia, 2016; 196p, ISBN 978-5-406-04805-4. (In Russian)

23. Mainardi, F. Fractional relaxation-oscillation and fractional diffusion-wave phenomena. Chaossolitons Fractals 1996, 7, 1461-1477. [CrossRef]

24. Tarasov, V.E. Fractional Dynamics: Applications of Fractional Calculus to Dynamics of Particles, Fields and Media; Springer: New York, NY, USA, 2010; 505p. [CrossRef]

25. Tarasov, V.E. The fractional oscillator as an open system. Open Phys. 2012, 10, 382-389. [CrossRef]

26. Li, M. Three classes of fractional oscillators. Symmetry 2018, 10, 40. [CrossRef]

27. Parovik, R. Mathematical modeling of linear fractional oscillators. Mathematics 2020, 8, 1879. [CrossRef]

28. Barro, R.J.; Sala-i-Martin, X.I. Economic Growth, 2nd ed.; The MIT Press: Cambridge, MA, USA; London, UK, 2003; 654p, ISBN-13 978-0262025539.

29. Romer, D. Advanced Macroeconomics, 3rd ed.; McGraw-Hill Companies: Boston, MA, USA, 2006; 678p, ISBN 978-0-07-287730-8.

30. Odibat, Z.M.; Shawagfeh, N.T. Generalized Taylor's formula. Appl. Math. Comput. 2007, 186, $286-293$. [CrossRef]

31. Haavelmo, T. A Study in the Theory of Economic Evolution; North-Holland: Amsterdam, The Netherlands, 1964; 114p. Available online: http://www.sv.uio.no/econ/english/research/networks/haavelmo-network/ publications/files/TH1954a.pdf (accessed on 12 December 2020).

Publisher's Note: MDPI stays neutral with regard to jurisdictional claims in published maps and institutional affiliations.

(C) 2020 by the author. Licensee MDPI, Basel, Switzerland. This article is an open access article distributed under the terms and conditions of the Creative Commons Attribution (CC BY) license (http://creativecommons.org/licenses/by/4.0/). 